\title{
Heat Transfer Characteristics and Reynolds Stress Budgets in Single-Rib Mounting Channel*
}

\author{
Takahiro MIURA $^{* *}$, Koji MATSUBARA ${ }^{* * *}$ and Atsushi SAKURAI ${ }^{* * *}$ \\ ${ }^{* *}$ Graduate School of Science and Technology, Niigata University, \\ 8050 Ikarashi 2-no-cho, Nishi-ku, Niigata 950-2181, Japan \\ E-mal: f09k008c@mail.cc.niigata-u.ac.jp \\ ***Department of Mechanical and Production Engineering, Niigata University, \\ 8050 Ikarashi 2-no-cho, Nishi-ku, Niigata 950-2181, Japan
}

\begin{abstract}
Heat transfer and fluid flow in a single-rib mounting channel were investigated by directly solving Navier-Stokes and energy equations. Flow and thermal fields were considered to be fully developed at the inlet of the channel, and the simulation was made for spatial advancement of turbulent heat transfer. Keeping the frictional Reynolds number, $R e_{\tau}$, at 150 , the rib height ratio was changed in four steps from $H / \delta=0.05$ to $H / \delta=0.4$. Computational results were confirmed to be nearly independent of grid meshes. In addition, numerical accuracy was confirmed through close agreement between computed mean pressure and the experiment by Yao et al. (1995). The numerical results revealed that the highest value of the mean Nusslet number was as large as 1.3 times the smooth surface consuming the same pumping power, and the local enhancement of heat transfer was correlated with the turbulence increase near the rib front and the reattachment point. According to the Reynolds stress budgets for $H / \delta=0.2$, there were mechanisms to induce powerful fluctuations: (1) Streamwise fluctuation was increased through production by flow deceleration in the upstream of the rib; (2) Redistribution to wall-normal and spanwise fluctuations was fortified by the fluid splattering to the rib front. Therefore, excellent performance of heat transfer was concluded to occur due to flow structures, which induce the strong disturbance near the rib front triggering smooth transition of the separated shear layer.
\end{abstract}

Key words: Turbulent Flow, Heat Transfer Enhancement, Pressure Drop, Direct Numerical Simulation, Rectangular Rib, Reynolds Stress Budget

\section{Introduction}

The wall roughness or the protrusions are convenient tools to destabilize wall-bounded flows. This type of device can be utilized for fluid control and augmentation of heat transfer in turbo-machineries, power generators, combustors and chemical reactors. Enhancement of inner-cooling in gas-turbine blades is one of applications. Optimization of the roughness or the turbulator is a key to achieve better performance in machineries of energy conversion and process engineering.

In addition to the practical importance of fully developed cases of periodic obstacles $^{(1)-(5)}$, the case of a single obstacle means a lot both scientifically and practically ${ }^{(6)-(10)}$. Suzuki et al. ${ }^{(6)}$ reported that the heat transfer increase and the friction decrease simultaneously occurred in a local spot of the turbulent boundary layer inserted by an cylinder. Yao et al..$^{(7)}$ visualized flow fields in a similar situation, and they argued about roller structures behind the obstacle, which caused the local dissimilarity between the heat 
transfer and the friction on the wall.

In spite of these efforts, there is ambiguity in the heat transfer enhancement by a single obstacle. At the present time, it is not evident if the overall heat transfer in the case with an obstacle can actually exceed the flat-plate case. The recent DNSs (Direct Numerical Simulations) for non-periodic cases ${ }^{(8)-(10)}$ did not resolve this question since they targeted on basic fluid mechanics not paying special attention to the heat transfer enhancement.

In the present study, a spatially advancing type of DNS is performed for turbulent air flows in a channel with a single rib mounted on the wall. The frictional Reynolds number is kept at 150 at the channel inlet, and the ratio of the rib height to the channel half width is changed in $0.05,0.1,0.2$ and 0.4 . The mean Nusselt number is analyzed through comparison with the flat-plate case showing the same pumping loss in order to verify the total merit of the single-rib augmentation. Mechanisms of heat transfer enhancement are examined through discussion of turbulence statistics of flow and temperature and the Reynolds stress budgets. An instantaneous flow is argued to compile information on turbulence structures related to transport process.

\section{Nomenclature}

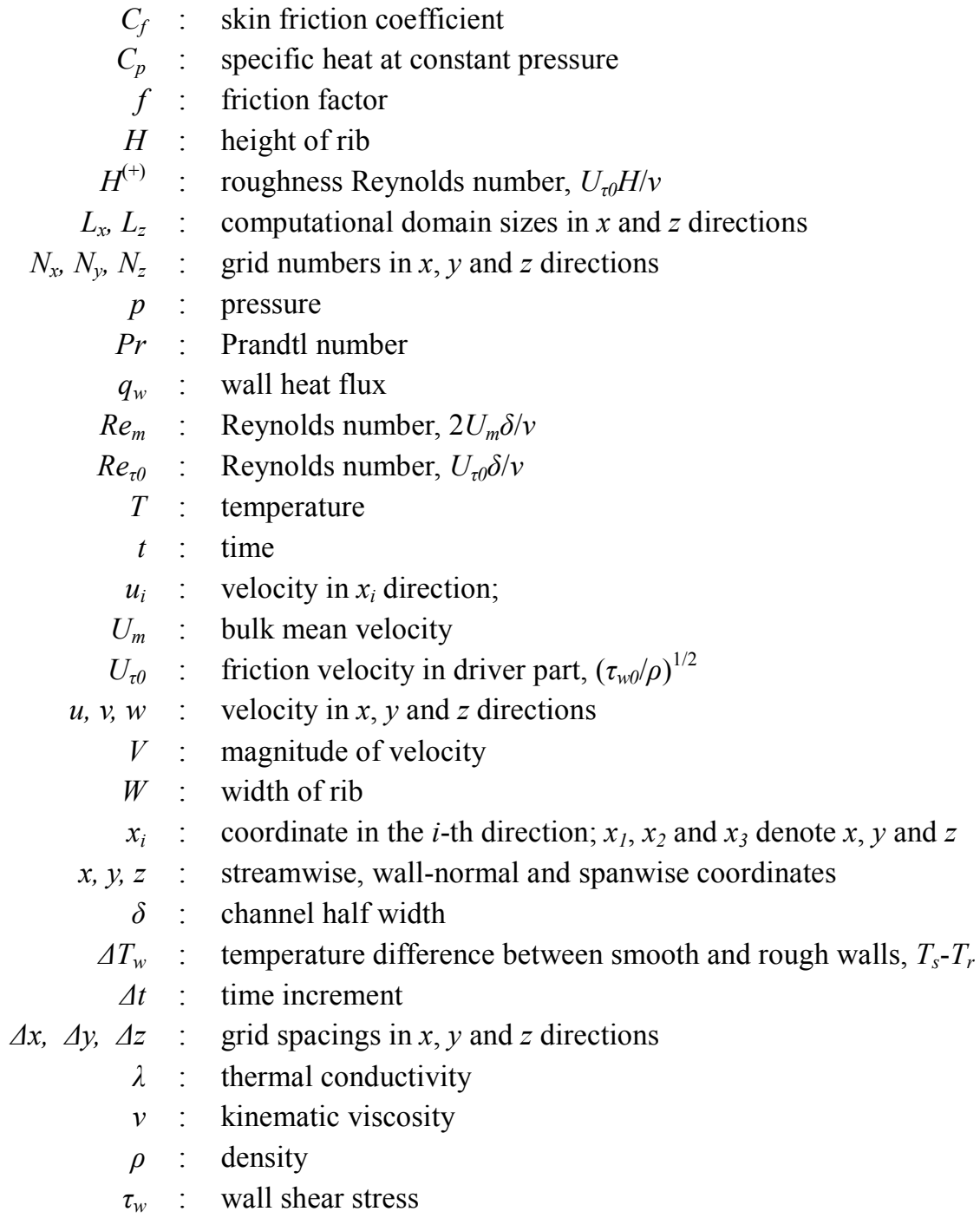

\section{Subscripts and Superscripts}

()$_{0} \quad$ : value in driver part or on inlet 

( ) : value on rough-wall side
( ) : value on smooth-wall side
( )' : fluctuating component
()$^{(+)}:$normalized by $U_{\tau 0}$ and $v$
$\overline{()}$ : ensemble averaged value

\section{Numerical Method}
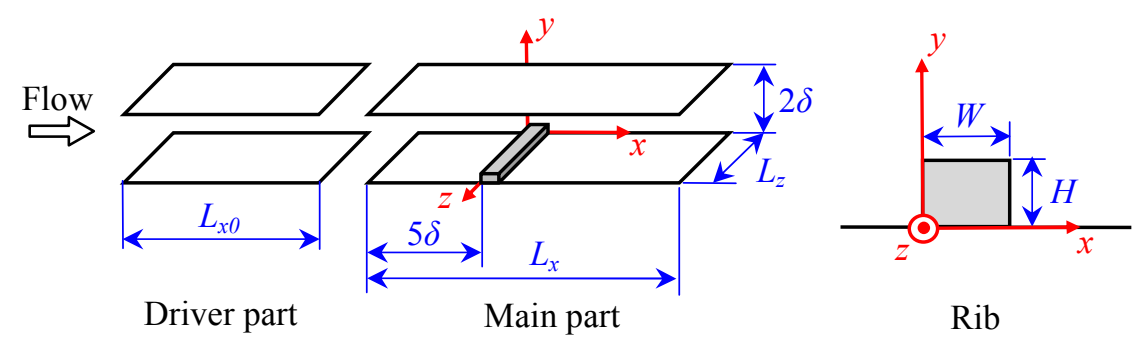

Fig. 1 Computational domain and coordinate system.

Figure 1 shows the computational domain and the coordinate system. A fully developed turbulent flow with thermal variation is assumed to enter a straight channel obstructed by one rectangular rib protruded from the wall. The inlet values for the ribbed channel are generated by the driver part, where a constant pressure gradient drives the fluid flow. Periodic boundary condition is employed in streamwise and spanwise directions of the driver part, and in the spanwise direction of the main part. In both of the driver and main parts, non slip condition is used on channel walls. Thermally, temperature of the lower walls and rib surfaces are kept at $\left(T-T_{r}\right) / \Delta T_{w}=0$, and that of the upper walls are fixed at $\left(T-T_{r}\right) / \Delta T_{w}=1$. At the exit of the main part, the convection out-flow condition ${ }^{(11)}$ is applied for flow and temperature fields.

The continuity, Navier-Stokes and energy equations for the incompressible fluid;

$$
\begin{aligned}
& \frac{\partial u_{j}}{\partial x_{j}}=0 \\
& \frac{\partial u_{i}}{\partial t}+u_{j} \frac{\partial u_{i}}{\partial x_{j}}=-\frac{1}{\rho} \frac{\partial p}{\partial x_{i}}+v \frac{\partial^{2} u_{i}}{\partial x_{j} \partial x_{j}} \\
& \frac{\partial T}{\partial t}+u_{j} \frac{\partial T}{\partial x_{j}}=\frac{\lambda}{\rho C_{p}} \frac{\partial^{2} T}{\partial x_{j} \partial x_{j}},
\end{aligned}
$$

are used as governing equations. As implied by Eq. (2), gravity effects are neglected. Time advancement of Eqs. (1) and (2) is made by the fractional step method ${ }^{(12)}$. In time splitting of Eqs. (2) and (3), the second-order Crank-Nicolson method is applied for the wall-normal second derivatives, and the second-order Adams-Bashforth method is used for other terms. The forth-order central difference ${ }^{(13),(14)}$ is utilized as spatial difference for convection and diffusion terms of Eqs. (2) and (3). In the driver part, the Poisson equation for the pressure is solved by the fast Fourier transformation (FFT) in the streamwise and spanwise directions and by the TDMA of compact difference for wall-normal derivatives. In the ribbed channel, the Poisson equation is computed by the FFT in the spanwise direction and by the SOR at each plane perpendicular to the spanwise axis.

Computational conditions are listed in Table 1. The rib height is changed in four steps ranging from $H / \delta=0.05$ to $H / \delta=0.4$. The Reynolds number based on the frictional velocity and the channel half width, $R e_{\tau 0}$, is set at 150 for $H / \delta=0.05,0.1,0.2,0.4$ (Cases 2, 
3a-3d, 4, and 5), and at 180 for $H / \delta=0.4$ (Case 1) which exactly corresponds to the experiment ${ }^{(7)}$. Air flow is assumed through the simulations, and the Prandtl number is fixed at 0.71 .

Figure 2 shows an example of grid arrangement. As shown in this figure, numerical grids are allocated finely to the rib walls, especially dense in upstream of the front surface of the rib. Such a grid arrangement is used in order to resolve the thin boundary layer in the impinging region without numerical oscillation. For $H / \delta=0.2$, four kinds of computation (Cases 3a-3d) are performed with using different grid meshes. Case 3a and Case $3 \mathrm{~b}$ are simulated by the largest computational volume with the spanwise length of $L_{z} / \delta=6.4$. In the former and the latter, grid numbers are $(128+320) \times 185 \times 256$ and $(64+320) \times 98 \times 128$, respectively. Case $3 \mathrm{c}$ is computed by the reduced domain, where the spanwise length is a half of Case $3 \mathrm{a}$ or $3 \mathrm{~b}\left(L_{z} / \delta=3.2\right)$, and grid resolution is $(64+320) \times 98$ $\mathrm{x}$ 64. Case $3 \mathrm{~d}$ is also computed by the reduced domain, having sparser grids in the streamwise direction than Case 3c. Figures 3 and 4 show distributions of the skin friction coefficient and the Nusselt number, respectively, for Cases 3a-3d. There is almost no essential difference occurring from changes in the domain size or the grid arrangement. It is thus suggested that the domain size is enough large with sufficient resolution of grids through Cases 3a-3d. As suggested in Table 2, simulations for Cases 1, 2, 4, and 5 are made by the reduced domain with grid resolution as fine as Case $3 b$ or $3 c$ to save the computational load.

Table 1 Computational conditions.

\begin{tabular}{cccccc}
\hline & Case 1 & Case 2 & Case 3a-3d & Case 4 & Case 5 \\
\hline$H / \delta$ & 0.4 & 0.4 & 0.2 & 0.1 & 0.05 \\
$W / \delta$ & 0.4 & 0.4 & 0.2 & 0.2 & 0.2 \\
$\operatorname{Re}_{\tau} 0$ & 180 & 150 & 150 & 150 & 150 \\
$H^{(+)}$ & 72 & 60 & 30 & 15 & 7.5 \\
$P r$ & 0.71 & 0.71 & 0.71 & 0.71 & 0.71 \\
\hline
\end{tabular}

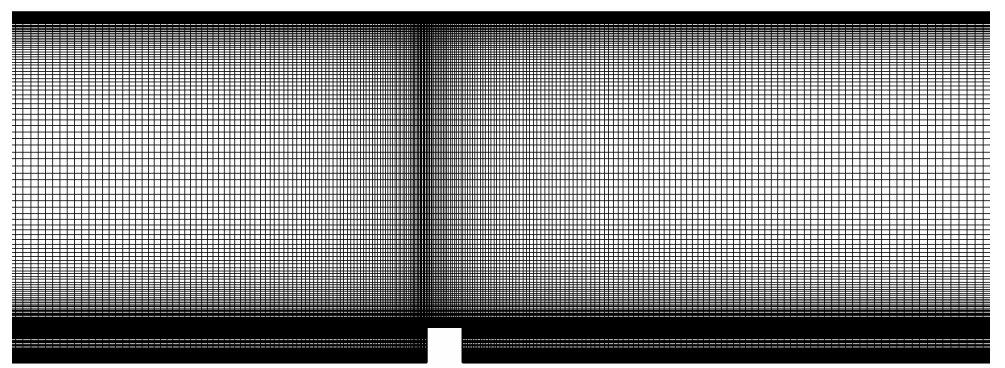

Fig. 2 Grid arrangement near rib.
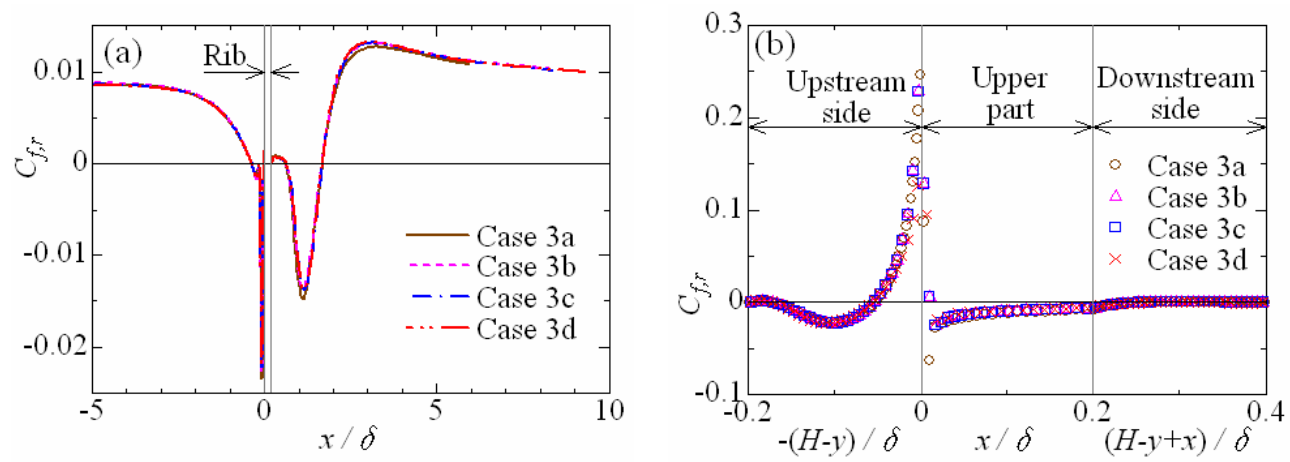

Fig. 3 Local skin friction coefficient: (a) bottom wall; (b) rib. 

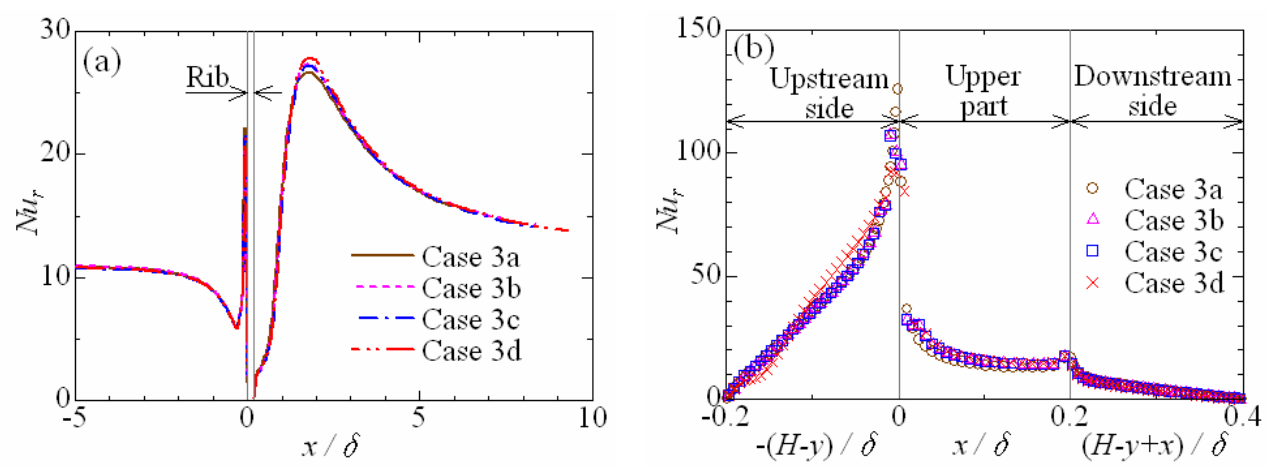

Fig. 4 Local Nusselt number: (a) bottom wall; (b) rib.

Table 2 Computational domain and grid arrangement.

\begin{tabular}{ccccc}
\hline & Case 1 & Case 2 & Case 4 & Case 5 \\
\hline$L_{x 0} / \delta$ & 8 & 8 & 8 & 8 \\
$L_{x} / \delta$ & 10.6 & 22.9 & 13.4 & 13.4 \\
$L_{z} / \delta$ & 3.2 & 3.2 & 3.2 & 3.2 \\
$N_{x 0}$ & 128 & 64 & 64 & 64 \\
$N_{x} \times N_{y} \times N_{z}$ & $320 \times 128 \times 128$ & $512 \times 128 \times 64$ & $320 \times 98 \times 64$ & $320 \times 98 \times 64$ \\
$\Delta x_{0}{ }^{(+)}$ & 11.3 & 18.8 & 18.8 & 18.8 \\
$\Delta x^{(+)}$ & $1.13-11.3$ & $0.938-14.1$ & $0.938-14.1$ & $0.938-14.1$ \\
$\Delta y^{(+)}$ & $1.13-7.34$ & $0.938-6.12$ & $0.938-10.0$ & $0.938-10.0$ \\
$\Delta z^{(+)}$ & 4.50 & 7.50 & 7.50 & 7.50 \\
$\Delta t^{(+)}$ & 0.00720 & 0.00600 & 0.0120 & 0.0120 \\
\hline \hline & Case $3 \mathrm{a}$ & Case $3 \mathrm{~b}$ & Case $3 \mathrm{c}$ & Case $3 \mathrm{~d}$ \\
\hline$L_{x 0} / \delta$ & 16 & 8 & 8 & 8 \\
$L_{x} / \delta$ & 11.0 & 13.4 & 13.4 & 14.3 \\
$L_{z} / \delta$ & 6.4 & 6.4 & 3.2 & 3.2 \\
$N_{x 0}$ & 128 & 64 & 64 & 64 \\
$N_{x} \times N_{y} \times N_{z}$ & $320 \times 185 \times 256$ & $320 \times 98 \times 128$ & $320 \times 98 \times 64$ & $320 \times 98 \times 64$ \\
$\Delta x_{0}{ }^{(+)}$ & 18.8 & 18.8 & 18.8 & 18.8 \\
$\Delta x^{(+)}$ & $0.938-9.38$ & $0.938-14.1$ & $0.938-14.1$ & $1.88-14.1$ \\
$\Delta y^{(+)}$ & $0.300-6.00$ & $0.938-10.0$ & $0.938-10.0$ & $0.938-10.0$ \\
$\Delta z^{(+)}$ & 3.75 & 7.50 & 7.50 & 7.50 \\
$\Delta t^{(+)}$ & 0.00600 & 0.0120 & 0.0120 & 0.0120 \\
\hline & & & &
\end{tabular}

\section{Results and Discussion}

\subsection{Pressure Loss and Heat Transfer}

Mean pressure distributions for $H / \delta=0.2$ and $H / \delta=0.4$ are shown in Fig. 5. In the figure, Case 1 for $H / \delta=0.4$ and $R e_{m}=5650$ is very close to the experiment for the same condition by Yao et al..$^{(7)}$, and validity of the numerical data is thus confirmed. In all the presented cases, there are pressure drops due to the blockage effect from the rib, and the mean values show transitional variation in the upstream and downstream region between $-5 \leqq x / H \leqq 30$.

Pressure loss and heat transfer in a channel are evaluated, respectively, by the apparent skin friction coefficient

$$
f=\frac{1}{\rho U_{m}{ }^{3}\left(x_{d}-x_{u}\right)}\left[\left.\int_{0}^{2 \delta}\left(\bar{p}+\frac{\rho \bar{V}^{2}}{2}\right) \bar{u} d y\right|_{x=x_{u}}-\left.\int_{0}^{2 \delta}\left(\bar{p}+\frac{\rho \bar{V}^{2}}{2}\right) \bar{u} d y\right|_{x=x_{d}}\right]
$$




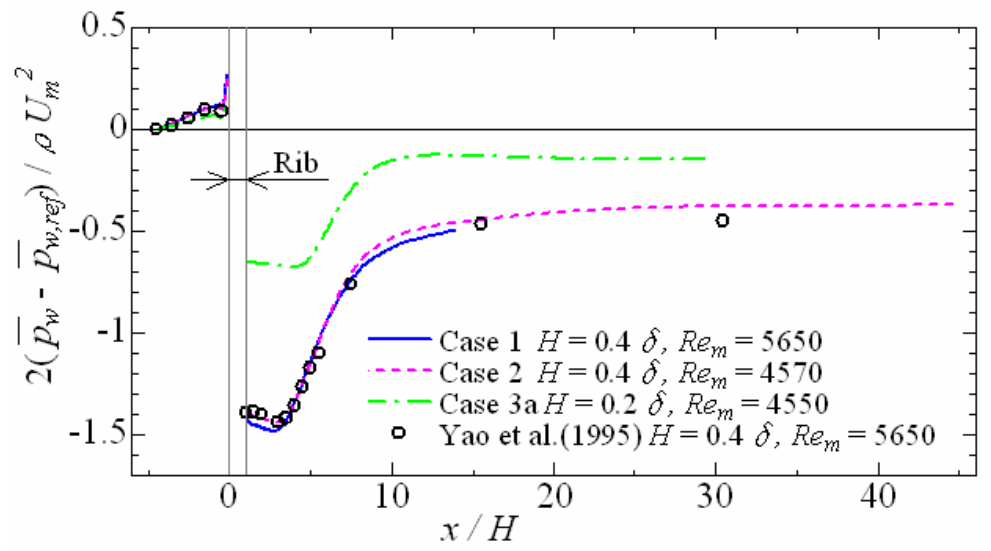

Fig. 5 Distribution of mean pressure on bottom wall $\left(\bar{p}_{w, r e f}\right.$ is pressure at $\left.x / H=-4.5\right)$.

and the mean Nusselt number

$$
\langle N u\rangle=\frac{4 \delta}{\lambda\left(T_{w}-T_{w, \text { other }}\right)\left(x_{d}-x_{u}\right)} \int_{x_{u}}^{x_{d}} q_{w} d x
$$

The reference planes are chosen as $x_{u}=-20 H$ and $x_{d}=40 H$, by which major part of the rib effects can be included in the parameters. Computed values from Eqs. (4) and (5) are shown in Figs. 6 and 7, respectively. The former presents experimental correlation for a smooth channel by Dean ${ }^{(15)}$

$$
f=0.073 \operatorname{Re}_{m}{ }^{-0.25}
$$

and the Blasius's law ${ }^{(16)}$

$$
f=0.079\left(2 R e_{m}\right)^{-0.25}
$$

and the latter indicates the correlation by Tsukahara et al. ${ }^{(17)}$

$$
N u=0.014 \operatorname{Re}_{m}^{0.8} \operatorname{Pr}^{0.5}
$$

In Fig. 6, the friction coefficient on the flat surface is doubled by the highest $\mathrm{rib}(H / \delta=0.4)$. However, in Fig. 7, even maximum of the mean Nusselt number does not reach twice of the flat-plate value.

In Fig. 8, the mean Nusselt number is plotted against $f^{1 / 3} R e_{m}$, which corresponds to cubic root of non-dimensional pumping power. The figure includes the correlation for flat-plate values deduced from Eqs. (6) and (8),

$$
N u=0.03 \operatorname{Pr}^{0.5}\left(f^{1 / 3} \operatorname{Re}_{m}\right)^{48 / 55}
$$

and that from Eqs. (7) and (8), 


$$
N u=0.0308 \operatorname{Pr}^{0.5}\left(f^{1 / 3} \operatorname{Re}_{m}\right)^{48 / 55} .
$$

In the figure, the mean Nusselt number on the ribbed wall is confirmed to exceed the smooth plate for the same pumping power. The enhancement ratio is 1.3 at maximum. Merit of the single-rib enhancement is thus validated for the case when the hydrodynamic loss is taken into consideration.

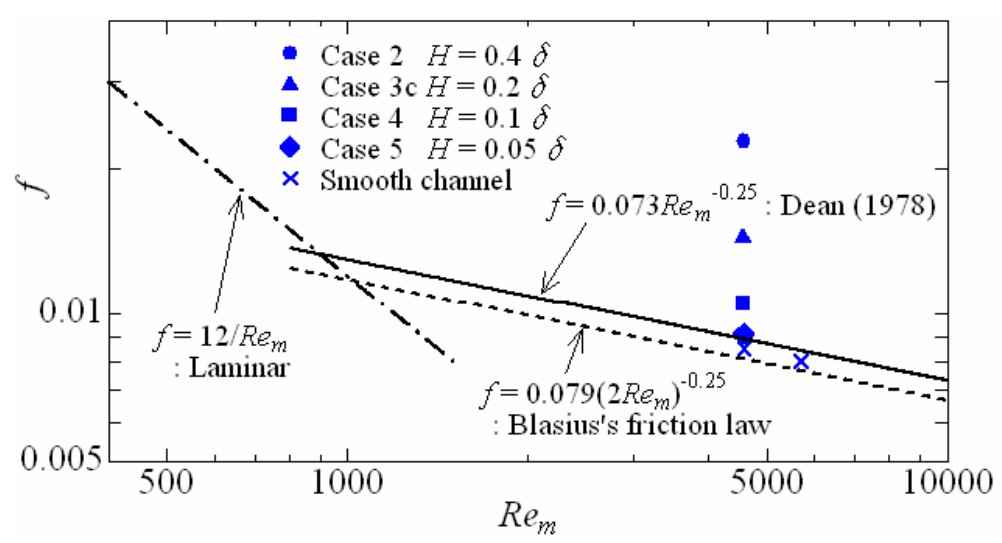

Fig. 6 Friction factor versus bulk Reynolds number.

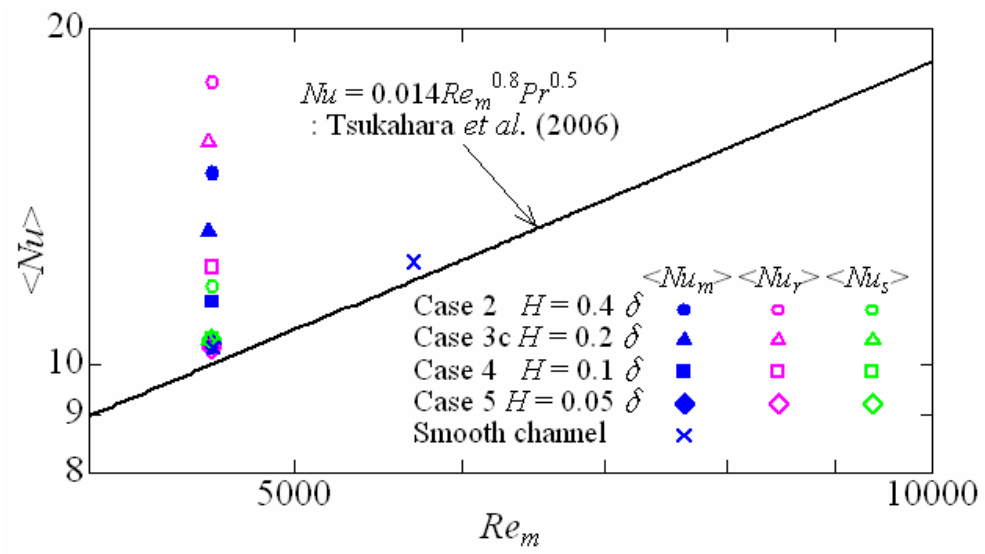

Fig. 7 Mean Nusselt number (on the two walls $<N u_{m}>$, on the rough-wall $<N u_{r}>$ and on the smooth-wall $<N u_{s}>$ ) versus bulk Reynolds number.

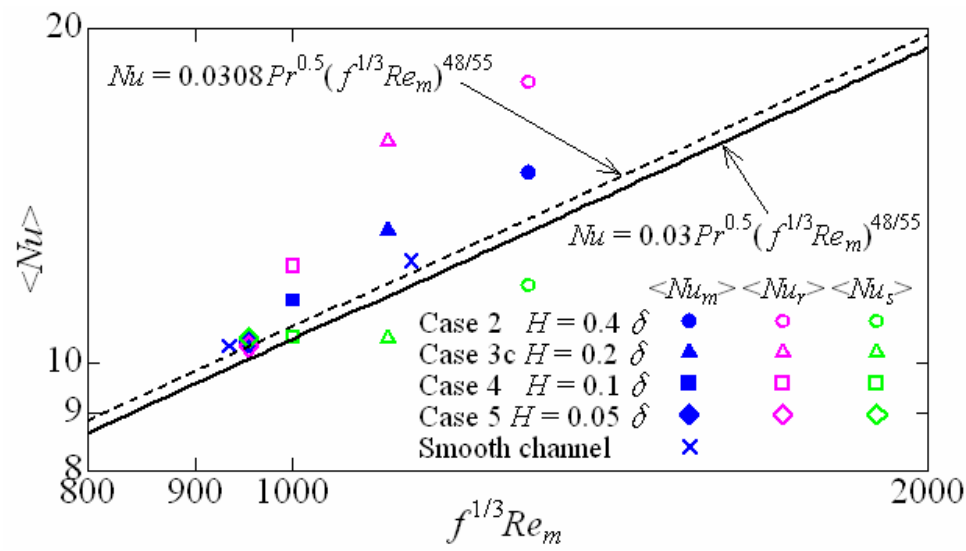

Fig. 8 Mean Nusslt number (on the two walls $\left\langle N u_{m}>\right.$, on the rough-wall $\left\langle N u_{r}\right\rangle$ and on the smooth-wall $<N u_{s}>$ ) versus pumping power. 


\subsection{Turbulence Statistics of Flow}

In the following, attention is paid to the results from Case $3 a(H / \delta=0.2)$, where heat transfer is preferably enhanced with increasing pressure loss penalty modestly as earlier mentioned.

Figure 9 shows mean streamlines together with colored gradation of the mean-velocity magnitude. In this figure, circulating flows exist next to the upstream and downstream surfaces of the rib. From numerical analysis, the separated shear layer from the rib is confirmed to reattach to $x / \delta=1.67$ on the wall, which is mostly correspond to the streamwise extent of the largest circulation as observed in the figure. In this case, the Nusselt number takes a peak at $x / \delta=1.75$ as suggested in Fig. 4, and this peak position is nearly coincident with the reattachment point.

The Reynolds normal stresses and the shear stress are shown in Figs. 10 and 11, respectively. In the downstream part of the rib, there are conspicuous increase in the Reynolds stresses, which is remarkable around $1.0<x / \delta<2.0$ prevailing in the upstream of the reattachment position. There are also high values of Reynolds stresses in the upstream of the rib, where the streamwise stress increases at $-0.5<x / \delta<0$ and others are large in close vicinity of the rib front. Increases in Reynolds stresses are correlated well with high values of Nusselt number in Fig. 4, and the heat transfer enhancement is suggested to occur due to the turbulent fluctuation.

The mean pressure and the root-mean-square of the fluctuation are shown in Figs. 12 and 13, respectively. In Fig. 12, mean pressure suddenly drops near the front edge of the rib, which implies an acute acceleration of the flow. In Fig. 13, pressure fluctuation is high in the downstream of the rib and in small spots near the front surface of the rib. As described later, such high fluctuations of pressure contribute the transport of Reynolds stresses through inter-component redistribution of turbulence energy and the pressure-assisting diffusion.

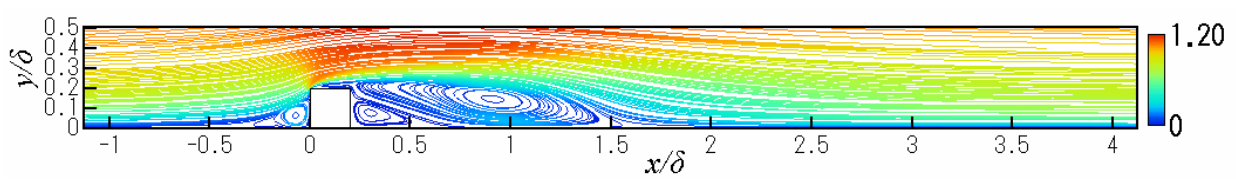

Fig. 9 Mean stream lines and contours of mean-velocity magnitude in Case 3a.
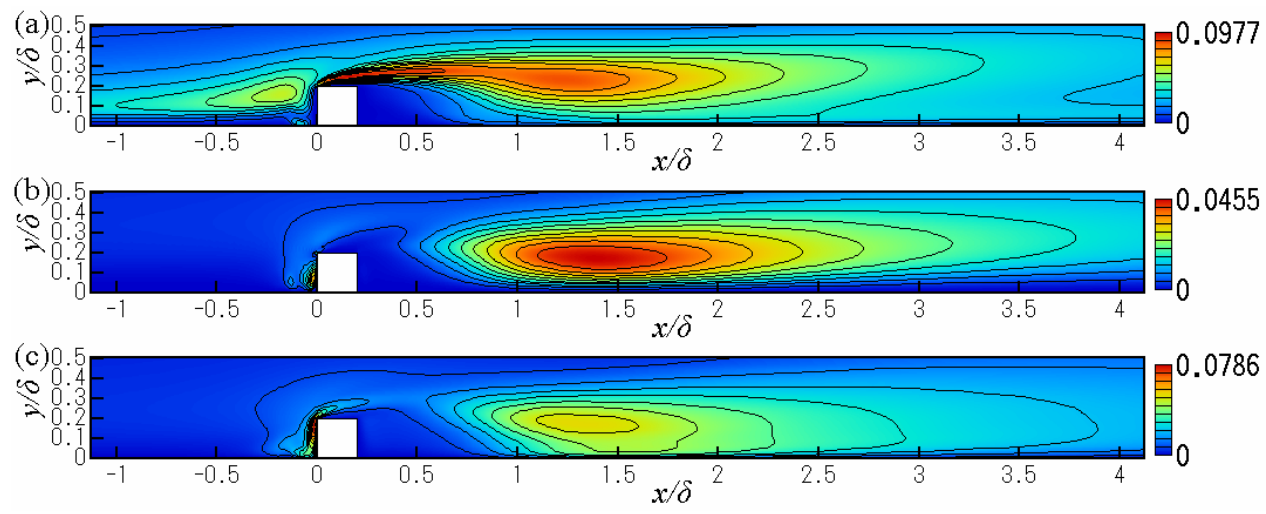

Fig. 10 Contours of Reynolds stress normalized by $U_{m}^{2}$ in Case 3a. (a) $\overline{u^{\prime} u^{\prime}}$; (b) $\overline{v^{\prime} v^{\prime}}$; (c) $\overline{w^{\prime} w^{\prime}}$.

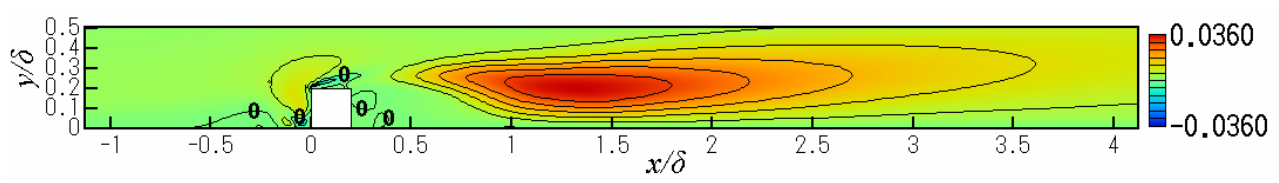

Fig. 11 Contours of Reynolds shear stress, $-\overline{u^{\prime} v^{\prime}}$, normalized by $U_{m}{ }^{2}$ in Case $3 \mathrm{a}$. 


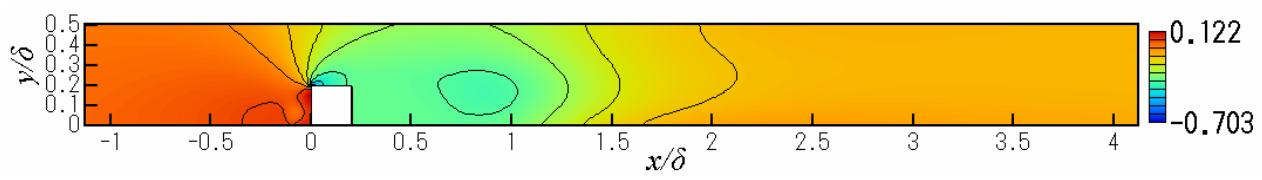

Fig. 12 Contours of mean pressure, $\left(\bar{p}-P_{0}\right) / \rho U_{m}{ }^{2}$, in Case 3a.

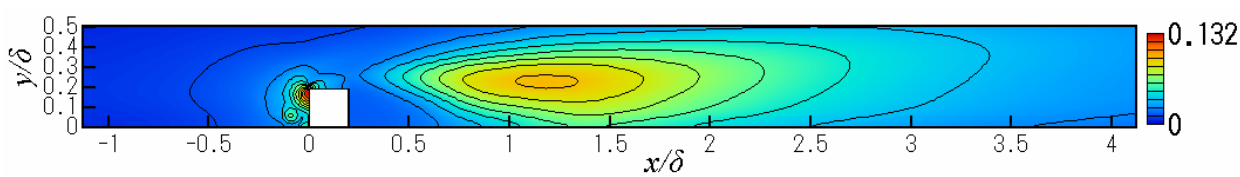

Fig. 13 Contours of root-mean-square pressure fluctuation normalized by $\rho U_{m}{ }^{2}$ in Case 3a.

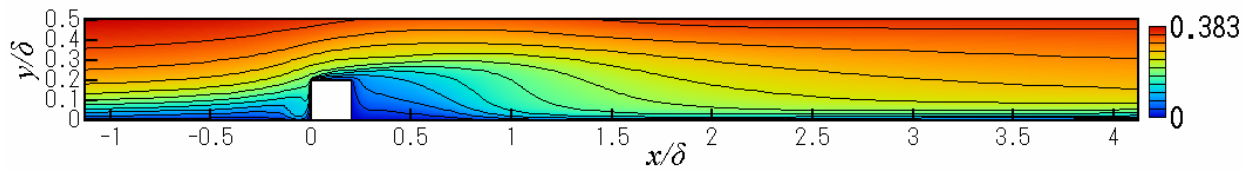

Fig. 14 Contours of mean temperature, $\left(\bar{T}-T_{r}\right) / \Delta T_{w}$, in Case 3a.
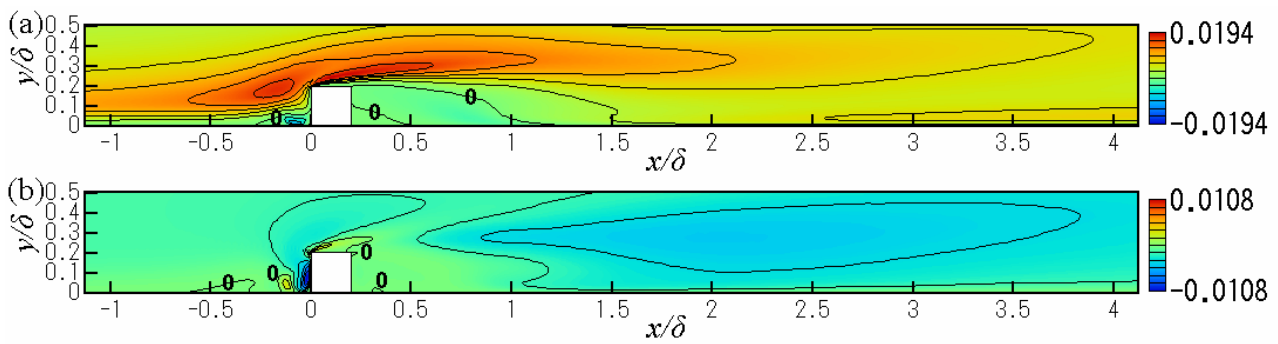

Fig. 15 Contours of turbulent heat flux normalized by $U_{m} \Delta T_{w}$ in Case 3a. (a) $\overline{u^{\prime} \theta^{\prime}}$; (b) $\overline{v^{\prime} \theta^{\prime}}$.

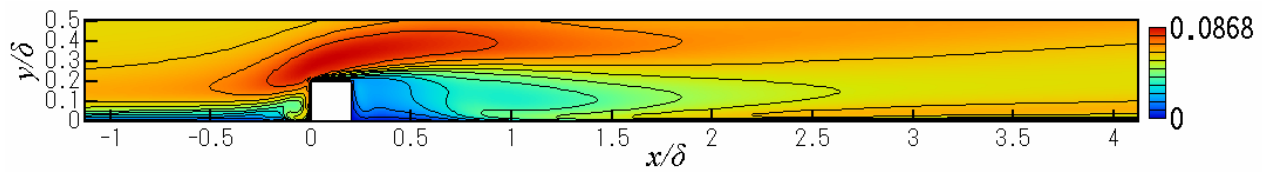

Fig. 16 Contours of root-mean-square temperature fluctuation normalized by $\Delta T_{w}$ in Case $3 \mathrm{a}$.

\subsection{Turbulence Statistics of Temperature}

Examination is given to the thermal field for the same case (Case 3 a for $H / \delta=0.2$ ) as treated in the previous section. Figure 14 shows the mean temperature. The thermal boundary layer is thin near the front surface of the rib and reattachment point $(x / \delta=1.67)$. Comparison of Fig. 14 with Figs. 4 and 9 reveals that the flow impingements on the walls lead to thin thermal layers enhancing heat transfer. The dense distributions of thermal contours near impinging regions contrast with sparse contours away from the wall, by which turbulent transport is implied to exist.

The streamwise and wall-normal turbulent heat fluxes are presented in Fig. 15. In the present study, $\overline{u^{\prime} \theta^{\prime}}>0$ and $\overline{v^{\prime} \theta^{\prime}}>0$ express turbulent transport in positive $x$ and $y$, respectively, while $\overline{u^{\prime} \theta^{\prime}}<0$ and $\overline{v^{\prime} \theta^{\prime}}<0$ mean transport in inverse directions. In most parts of the channel, $\overline{v^{\prime} \theta^{\prime}}$ shows negative values, and the turbulent heat flux is suggested to activate the heat travelling from the hotter (upper) to cooler (lower) walls. Similarly, positive values of $\overline{u^{\prime} \theta^{\prime}}$ near the rib front and its negative values near the back surface are consistent with heat directing to the rib surfaces. However, turbulent heat fluxes act to deteriorate the heat flow in small portions near the lower half of the rib's front surface 
$\left(\overline{u^{\prime} \theta^{\prime}}<0\right.$, Fig. 15(a)), its top surface $\left(\overline{v^{\prime} \theta^{\prime}}>0\right.$, Fig. 15(b)) and the channel wall just upstream of the rib front $\left(\overline{v^{\prime} \theta^{\prime}}>0\right.$, Fig. 15(b)). From Fig. 9, it is confirmed that such inverse contributions of turbulent heat flux tend to accompany with the separation of wall-bounded flow. Therefore, inverse contribution of turbulent motion is thought to occur in relation with bending of stream lines, which can inversely correlate flow and thermal fluctuations due to excessive convection of scalar.

Intensity of temperature fluctuation is displayed in Fig. 16. Temperature fluctuation is high around the rib's top surface extending between $-0.5<x / \delta<1.5$. This extension is broader than velocity fluctuation, $\overline{u^{\prime} u^{\prime}}$. This is because the thermal boundary condition adopted in this paper has the mean thermal gradient over the channel width and strong thermal fluctuation is assisted to occur through production by mean thermal gradient ${ }^{(18)}$.

\subsection{Budgets of Reynolds Stress}

Transport equations of Reynolds stresses can be written as follow:

$$
\frac{\partial \overline{u_{i}^{\prime} u_{j}^{\prime}}}{\partial t}+\bar{u}_{k} \frac{\partial \overline{u_{i}^{\prime} u_{j}^{\prime}}}{\partial x_{k}}=D_{i j}+P_{i j}+\Phi_{i j}+\varepsilon_{i j}
$$

Diffusion; $D_{i j}=\frac{\partial}{\partial x_{k}}\left\{v \frac{\partial \overline{u_{i} u_{j}}}{\partial x_{k}}-\overline{u_{i}^{\prime} u_{j}^{\prime} u_{k}^{\prime}}-\left(\overline{\frac{p u_{j}^{\prime}}{\rho}} \delta_{i k}+\frac{\overline{p u_{i}^{\prime}}}{\rho} \delta_{j k}\right)\right\}$;

Production; $P_{i j}=-\overline{u_{j}{ }^{\prime} u_{k}{ }^{\prime}} \frac{\partial \bar{u}_{i}}{\partial x_{k}}-\overline{u_{i}{ }^{\prime} u_{k}{ }^{\prime}} \frac{\partial \bar{u}_{j}}{\partial x_{k}}$;

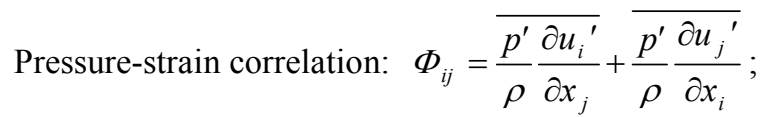

Dissipation; $\varepsilon_{i j}=-2 v \overline{\frac{\partial u_{i}{ }^{\prime}}{\partial x_{k}} \frac{\partial u_{j}{ }^{\prime}}{\partial x_{k}}}$.

In this section, budgets of Eq. (11) are presented for the Case $3 \mathrm{a}(H / \delta=0.2)$ similarly to sections 3.2 and 3.3. Figure 17 shows budgets in the driver part. As explained in the earlier work ${ }^{(19)}$, budgets in the no-rib case show that redistribution of energy goes from $\overline{u^{\prime} u^{\prime}}$ to $\overline{v^{\prime} v^{\prime}}$ and $\overline{w^{\prime} w^{\prime}}$ through the pressure-strain correlation being finally dissipated to heat, and $\overline{u^{\prime} v^{\prime}}$ disappears through another role of the pressure-strain correlation, namely destruction of the correlation. In all the budgets, diffusions are not important away from the wall, and the transport processes are in near local-equilibrium in major parts of the channel.

The sampling planes for Reynolds-stress budgets in the ribbed channel are indicated in Fig. 18. Figure 19 presents the budgets at $x / \delta=-1.0$ in the ribbed channel. In Fig. 19, budgets are similar to the driver part (Fig. 17), but each term is exaggerated. Further decomposition of production of $\overline{u^{\prime} u^{\prime}}$ revealed that the major contribution was made by an increase of $-2 \overline{u^{\prime} u^{\prime} \partial u} / \partial x$ rather than $-2 \overline{u^{\prime} v^{\prime}} \partial \bar{u} / \partial y$, and the exaggeration was suggested to occur due to the flow deceleration. In the production of $\overline{u^{\prime} v^{\prime}}$, increase of $-2 \overline{u^{\prime} u^{\prime}} \partial \bar{v} / \partial x$ was the most important among increases of $-2 \overline{u^{\prime} v^{\prime}} \partial \bar{u} / \partial x,-2 \overline{u^{\prime} u^{\prime}} \partial \bar{v} / \partial x$, $-2 \overline{v^{\prime} v^{\prime}} \partial \bar{u} / \partial y$ and $-2 \overline{u^{\prime} v^{\prime}} \partial \bar{v} / \partial y$, and it was suggested that displacement of mean flow increased the production.

Figure 20 shows budgets at $x / \delta=-0.12$, which is closer to the rib front. This plane still shows the essence of transport structures inherited from the driver part: redistribution occurs from $\overline{u^{\prime} u^{\prime}}$ to $\overline{v^{\prime} v^{\prime}}$ and $\overline{w^{\prime} w^{\prime}} ; \overline{u^{\prime} v^{\prime}}$ is destructed by the pressure-strain correlation. However, each term of budgets is more complex than the far-field due to the circulating flow neighboring the rib front. The diffusion terms (turbulent diffusion, 

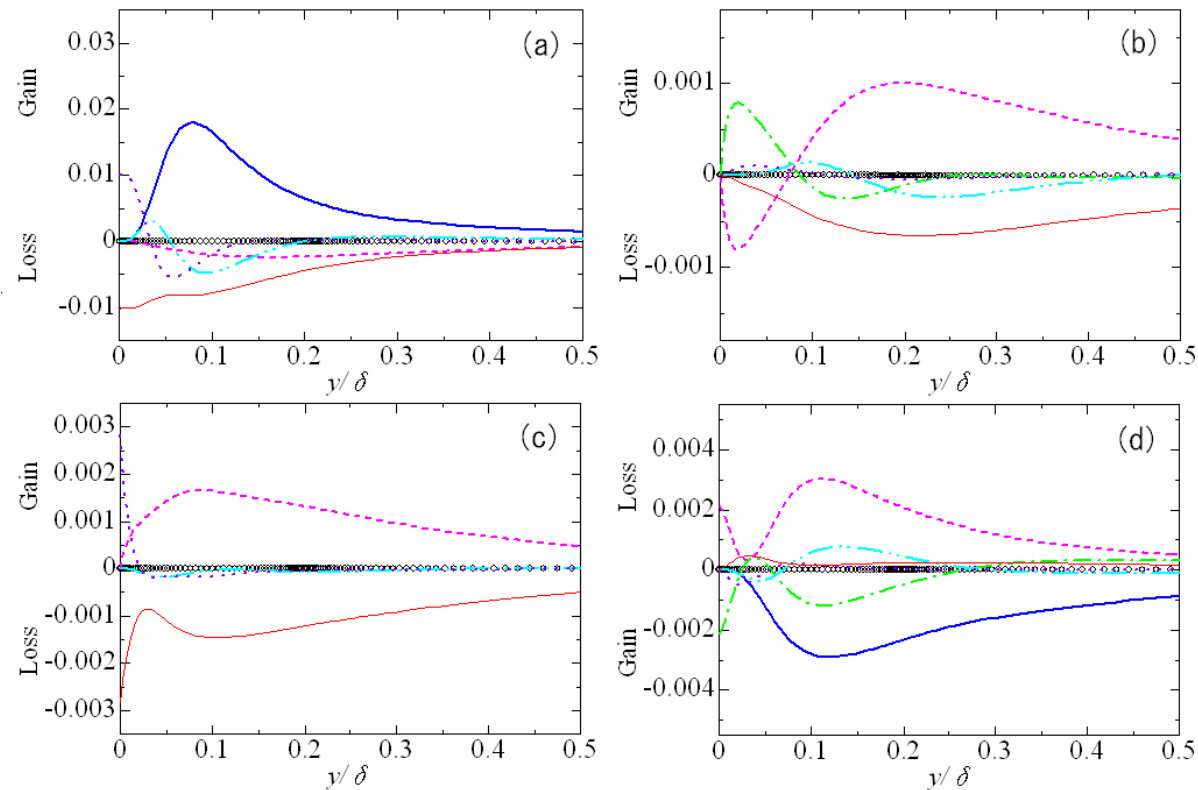

Fig. 17 Reynolds stress budgets normalized by $U_{m}{ }^{3} / \delta$ in Case 3a at driver part: —- production; -...--, pressure strain; -.., pressure diffusion; $-\cdots-$, turbulent diffusion; $\cdots .$. , viscous diffusion; dissipation; o, residual. (a) $\overline{u^{\prime} u^{\prime}}$; (b) $\overline{v^{\prime} v^{\prime}}$; (c) $\overline{w^{\prime} w^{\prime}}$; (d) $\overline{u^{\prime} v^{\prime}}$.

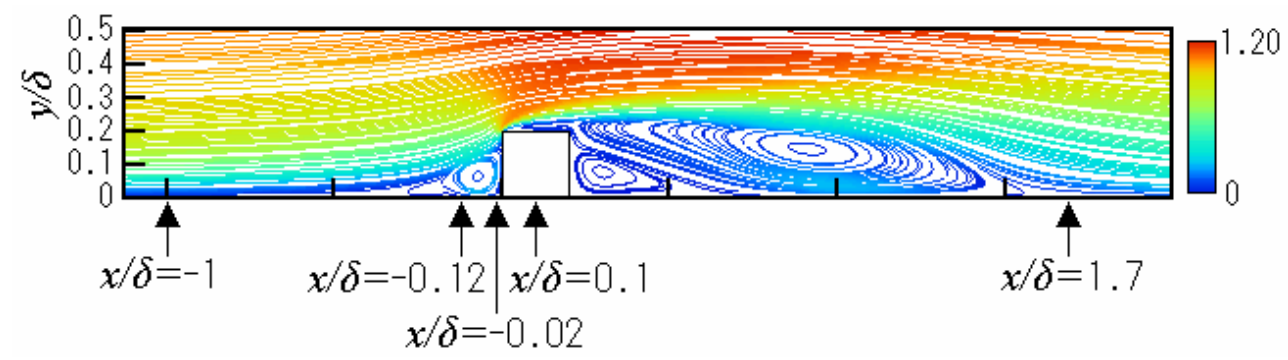

Fig. 18 Sampling position for Reynolds-stress budgets.
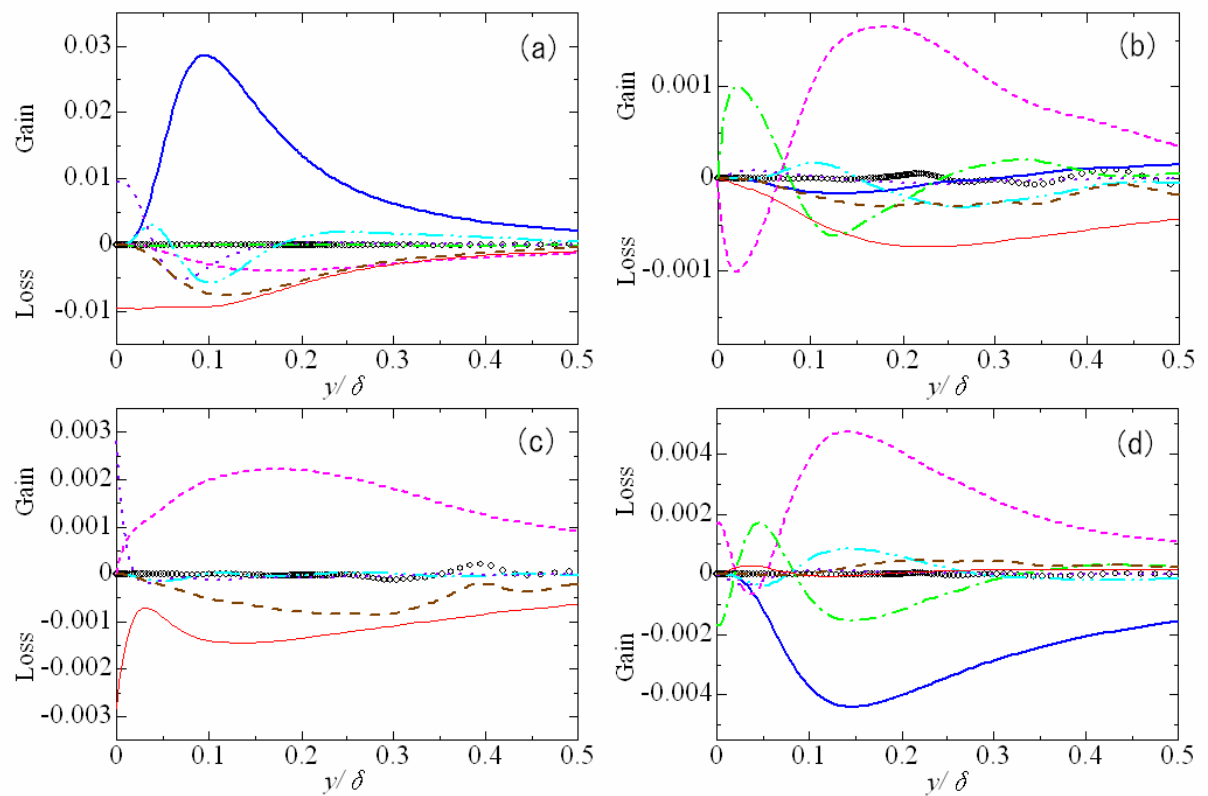

Fig. 19 Reynolds stress budgets normalized by $U_{m}{ }^{3} / \delta$ in Case 3 a at $x / \delta=-1$ : , production; -..-.pressure strain; - ..., pressure diffusion;..-- , turbulent diffusion; $\ldots .$. , viscous diffusion; —_ dissipation; - --, convection; 。, residual. (a) $\overline{u^{\prime} u^{\prime}}$; (b) $\overline{v^{\prime} v^{\prime}}$; (c) $\overline{w^{\prime} w^{\prime}}$; (d) $\overline{u^{\prime} v^{\prime}}$. 
pressure diffusion and viscous diffusion) are remarkable, and spatial transport is thus strong near the rib surface. In most parts of normal-stress budgets, diffusion terms or convections are larger than dissipation, and transport mechanisms are far from the local equilibrium.

Budgets at $x / \delta=-0.02$ are shown in Fig. 21. In the figure, pressure-strain correlations are huge in budgets of normal stresses, and the flow splattering to the front wall is suggested to promote the redistribution. Negative values in the production of $\overline{u^{\prime} u^{\prime}}$ and those in $\overline{v^{\prime} v^{\prime}}$ were confirmed to occur due to the flow acceleration. This acceleration was thought to occur based on the flow contraction near the rib corner and the impingement to the rib front. In all the budgets, diffusions or convection are important compared with the driver part, and the spatial transport is thus signified near the vertical wall.

In Fig. 22, budgets at $x / \delta=0.1$ are depicted. At this plane, gain by the production and loss by the convection are important in the budget of $\overline{u^{\prime} u^{\prime}}$. Pressure-strain correlation is small in the budgets of normal stresses, and the redistribution is shown to be minor.
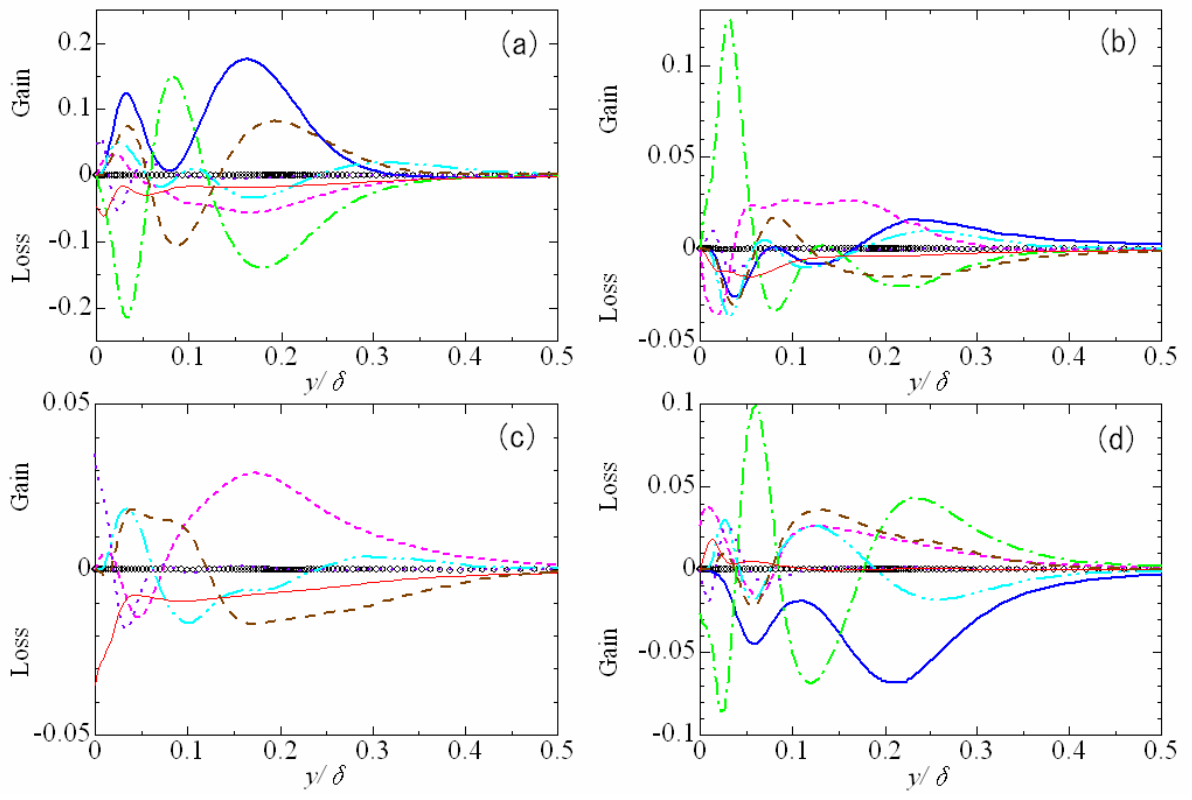

Fig.20 Reynolds stress budgets at $x / \delta=-0.12$ with the same captions as Fig. 19 .
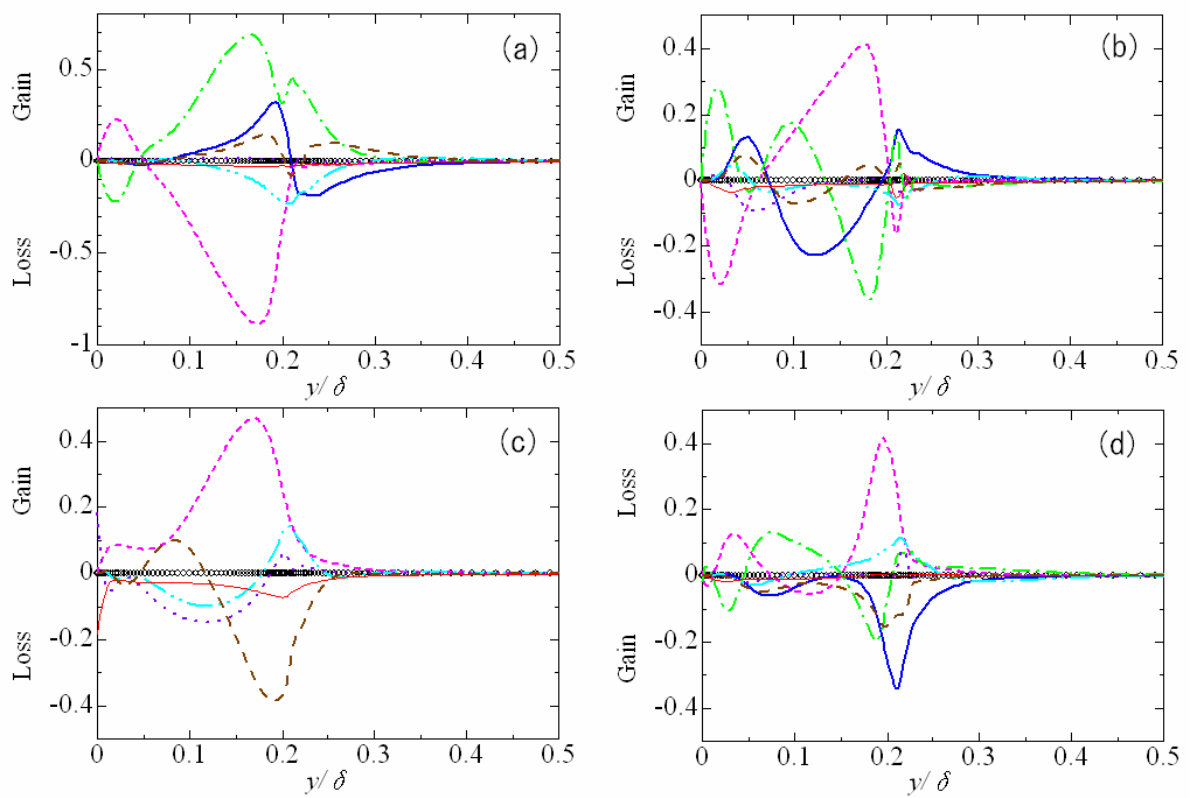

Fig.21 Reynolds stress budgets at $x / \delta=-0.02$ with the same captions as Fig. 19. 
In budgets of $\overline{v^{\prime} v^{\prime}}$ and $\overline{w^{\prime} w^{\prime}}$, the convection and diffusions are important, and diffusions transport stresses away from the wall. These are consistent with modest values of $\overline{v^{\prime} v^{\prime}}$ and $\overline{w^{\prime} w^{\prime}}$ near the top wall of the rib compared with $\overline{u^{\prime} u^{\prime}}$ as shown in Fig. 10.

Figure 23 shows the plane near the reattachment point, namely $x / \delta=1.7$. Near the reattachment point, redistribution occurs in normal stresses and contributions from turbulent diffusion are conspicuous in budget of $\overline{v^{\prime} v^{\prime}}$. Production of $\overline{v^{\prime} v^{\prime}}$ is high near the wall due to the deceleration of wall-normal velocity, and this contributes the high fluctuation which enhances scalar transport. There are remarkable contributions from pressure-strain correlation and pressure diffusion of $\overline{v^{\prime} v^{\prime}}$, and contributions from those of $\overline{u^{\prime} v^{\prime}}$ near the wall. Accordingly, splattering effects are suggested to occur due to the reattachment. These trends are similarly observed in cases of periodic fences ${ }^{(8)}$ and the backward step ${ }^{(11)}$, and the transport processes near the reattachment point are analogous in separated flows.
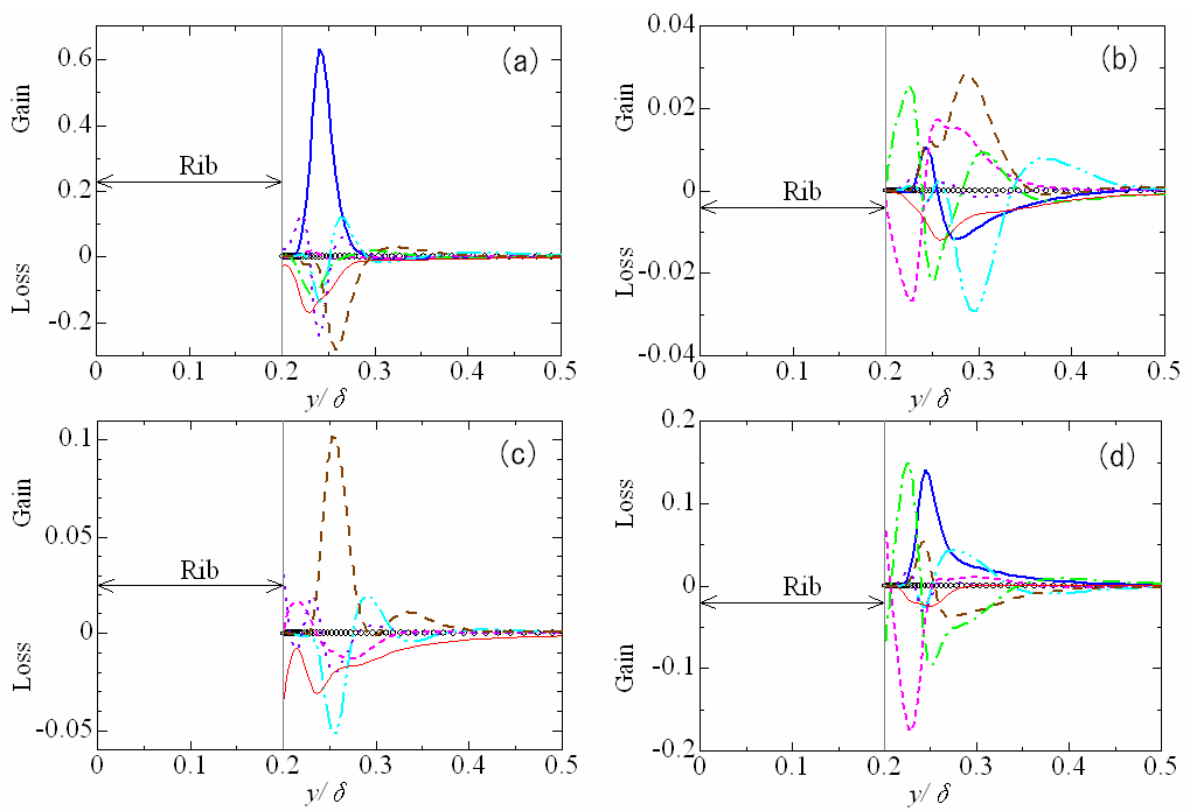

Fig.22 Reynolds stress budgets at $x / \delta=0.1$ with the same captions as Fig. 19.
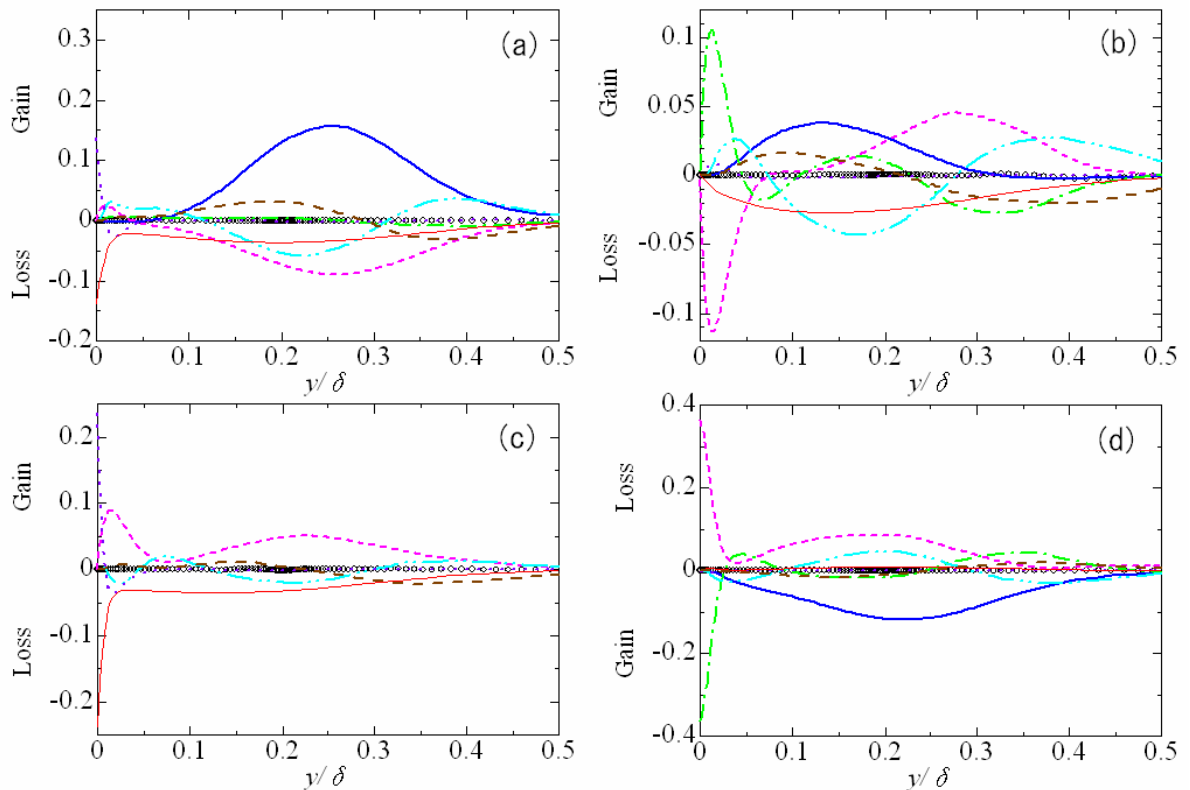

Fig. 23 Reynolds stress budgets at $x / \delta=1.7$ with the same captions as Fig. 19 . 


\subsection{Structures of Instantaneous Field}

An instantaneous flow is examined in order to understand turbulence structures related to the transport process. In Fig. 24, vortices are visualized by iso-surfaces of the second invariant for the deformation tensor

$$
Q=-\frac{1}{2} \frac{\partial u_{i}}{\partial x_{j}} \frac{\partial u_{j}}{\partial x_{i}}
$$

where the iso-surfaces are colored by

$$
Q_{z}=-\frac{1}{2}\left\{\left(\frac{\partial u}{\partial x}\right)^{2}+2 \frac{\partial u}{\partial y} \frac{\partial v}{\partial x}+\left(\frac{\partial v}{\partial y}\right)^{2}\right\}
$$

to detect the spanwise rotation. The figure includes contours of streamwise velocity fluctuation. In the upstream of the rib, there are stripes of alternating low-speed streaks and high-speed islands, and streaky structures are branched as approaching the rib wall. The branching of structures is implied to occur due to flow deceleration, and this is suggested to correspond to turbulence enhancement described in the previous section. Near the front surface of the rib, longitudinal structures are rooted to streaks and stretched to the upper-downstream direction, which implies energy redistribution occurring from streamwise to secondary directions through longitudinal vortices. There are longitudinal and spanwise structures near the top wall of the rib, and three-dimensional disturbance is suggested to occur in the mean flow. Behind the rib, instability in the shear layer is highly complex and fully three-dimensional, and three-dimensionality near the rib corner is suggested to trigger smooth transition.

Comparison between Figs. 24 and 4 reveals that high performance of heat transfer can be attributed to hydrodynamic mechanisms leading to strong fluctuation. Turbulence near the front surface of the rib is intensified due to flow deceleration, and three-dimensionality near the rib corner induces fast transition of separated shear layer. These structures are thought to induce powerful fluctuations near the wall, and activated transport of scalar is suggested to cause high values of Nusselt number.

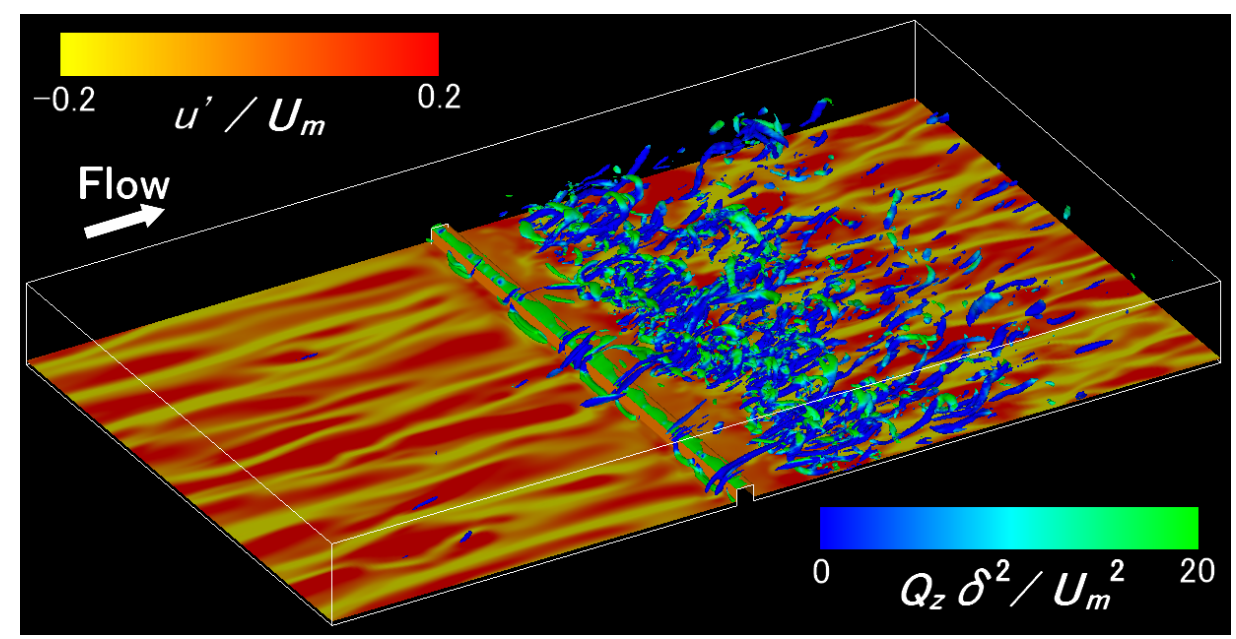

Fig. 24 Iso-surfaces of the second-invariant $\left(Q \delta^{2} / U_{m}{ }^{2}=20\right)$ with $Q_{z}$ and contours of streamwise velocity fluctuation at $y / \delta \fallingdotseq 0.05$ in Case 3 a. 


\section{Conclusion}

Direct numerical simulation was performed for air flows and related heat transfer in a channel with a single rib attached to the channel wall. The rib height was changed at $H / \delta=0.05,0.10,0.20,0.40$ for the frictional Reynolds number, $R e_{\tau}$, fixed at 150 . Conclusions thus extracted are as follow.

- For cases of $H / \delta=0.2$, four kinds of computation were made with changing spanwise length of computational domain and grid arrangement. Computational results from these simulations show almost no difference, and there was essentially no dependence on domain size and grids. In addition, in the case of the highest rib $(H / \delta=0.4)$, there was close agreement of the mean pressure between the computation and the existing experiment.

- The mean Nusselt number on the ribbed wall was about 1.3 times as large as the smooth wall for the same pumping power. Single rib mounted on the wall was thus meaningful as a heat-transfer-enhancement technique.

- The Reynolds normal stress was high near the front surface of the rib and around the reattachment region, which was well correlated with high values of the Nusselt number. The streamwise and wall-normal turbulent heat fluxes were mostly consistent with enhancing heat transfer on the wall.

- Budgets of Reynolds stresses revealed transport mechanisms: (1) The flow deceleration in the upstream of the rib results in the enhancement of streamwise normal stress; (2) Near the front surface of the rib, redistribution from streamwise to wall-normal and spanwise stresses were large due to spattering effect; (3) Near the reattachment point, production of wall-normal stress is high, and this enhances fluctuation by which heat transport is activated.

- Instantaneous iso-surfaces of second invariant for deformation tensor and contours of streamwise fluctuation revealed: (1) The low-speed streaks branched as the flow approached the rib front surface, which was implied to enhance turbulence through accumulating fluctuations; (2) Near the leading edge of the rib, longitudinal eddies resulted in three-dimensional disturbance of the shear layer, corresponding to activation of redistribution; (3) In the downstream of the rib, there was highly three-dimensional turbulence in the separated shear layer.

- Comparison between an instantaneous flow and distributions of the Nusselt number indicated that streamwise fluctuation near the rib's front surface and fully developed turbulence in the separated shear layer are correlated well with high values of the Nusselt number. Therefore, high performance of heat transfer in the ribbed channel is attributed to the mechanisms to induce strong fluctuation.

\section{References}

(1) Lewis, M. J., Optimising the thermohydraulic performance of rough surfaces, International Journal of Heat and Mass Transfer, Vol. 18 (1975), pp.1243-1248.

(2) Miyake, Y., Tsujimoto, K. and Nakaji, M., Direct numerical simulation of rough-wall heat transfer in a turbulent channel flow, International Journal of Heat and Fluid Flow, Vol. 22 (2001), pp.237-244.

(3) Leonardi, S., Orlandi, P., Smalley, R. J., Djenidi, L. and Antonia, R. A., Direct numerical simulations of turbulent channel flow with transverse square bars on one wall, Journal of Fluid Mechanics, Vol. 491 (2003), pp.229-238.

(4) Leonardi, S., Orlandi, P., Djenidi, L. and Antonia, R. A., Structure of turbulent channel flow with square bars on one wall, International Journal of Heat and Fluid Flow, Vol. 25 (2004), pp.384-392. 
(5) Nagano, Y., Hattori, H. and Houra, T., DNS of velocity and thermal fields in turbulent channel flow with transverse-rib roughness, International Journal of Heat and Fluid Flow, Vol. 25 (2004), pp.393-403.

(6) Suzuki, H., Suzuki, K. and Sato, T., Dissimilarity between heat and momentum transfer in a turbulent boundary layer disturbed by a cylinder, International Journal of Heat and Mass Transfer, Vol. 31 (1988), pp.259-265.

(7) Yao, M., Nakatani, M. and Suzuki, K., Flow visualization and heat transfer experiments in a turbulent channel flow obstructed with an inserted square rod, International Journal of Heat and Fluid Flow, Vol. 16 (1995), pp.389-397.

(8) Makino, S., Iwamoto, K. and Kawamura, H., Turbulent structures and statistics in turbulent channel flow with two-dimensional slits, International Journal of Heat and Fluid Flow, Vol. 29 (2008), pp.602-611.

(9) Orellano, A. and Wengle, H., Numerical simulation (DNS and LES) of manipulated turbulent boundary layer flow over a surface-mounted fence, European Journal of Mechanics, B/Fluids, Vol. 19 (2000), pp.765-788.

(10) Yakhot, A., Liu, H. and Nikitin, N., Turbulent flow around a wall-mounted cube: A direct numerical simulation, International Journal of Heat and Fluid Flow, Vol. 27 (2006), pp.994-1009.

(11) Le, H., Moin, P. and Kim, J., Direct numerical simulation of turbulent flow over a backward-facing step, Journal of Fluid Mechanics, Vol. 330 (1997), pp.349-374.

(12) Kim, J. and Moin, P., Application of a fractional step method to incompressible Navier-Stokes Equations, Journal of Computational Physics, Vol. 59 (1985), pp.308-323.

(13) Matsubara, K., Kobayashi, M. and Maekawa, H., Direct numerical simulation of a turbulent channel flow with a linear spanwise mean temperature gradient, International Journal of Heat and Mass Transfer, Vol. 41 (1988), pp.3627-3634.

(14) Matsubara, K., Kobayashi, M., Sakai, T. and Suto, H., A study on spanwise heat transfer in a turbulent channel flow -eduction of coherent structures by a conditional sampling technique-, International Journal of Heat and Fluid Flow, Vol. 22 (2001), pp.213-219.

(15) Dean, R. B., Reynolds number dependence of skin friction and other bulk flow variables in two-dimensional rectangular duct flow, Transactions of the ASME, Journal of Fluids Engineering, Vol. 100 (1978), pp.215-223.

(16) Bejan, A., Convection heat transfer, 2nd edition, (1995), John Wiley and Sons, Inc.

(17) Tsukahara, T., Iwamoto, K., Kawamura, H. and Takeda, T., DNS of heat transfer in a transitional channel flow accompanied by a turbulent puff-like structure, Proceedings of 5th International Symposium on Turbulence, Heat and Mass Transfer, (2006), pp.193-196.

(18) Matsubara, K., Matsui, A., Miura, T., Sakurai, A., Suto, H. and Kawai, K., A spatially advancing turbulent flow and heat transfer in a curved channel, Heat Transfer-Asian Research, Vol. 39 (2010), pp.14-26.

(19) Mansour, N. N., Kim, J. and Moin, P., Reynolds-stress and dissipation-rate budgets in a turbulent channel flow, Journal of Fluid Mechanics, Vol. 194 (1988), pp.15-44. 